\title{
EARLY NEAR-INFRARED OBSERVATIONS OF SN 1993J
}

\author{
K. Matthews, ${ }^{1}$ G. Neugebauer, ${ }^{1}$ L. Armus,${ }^{2}$ And B. T. Soifer ${ }^{1,2}$ \\ Received 2001 September 7; accepted 2001 November 6
}

\begin{abstract}
Photometric observations in the near-infrared atmospheric windows from 1.25 to $3.7 \mu \mathrm{m}$ and spectrographic observations from 1.2 to $2.4 \mu \mathrm{m}$ are presented of supernova SN 1993J for 250 days after the outburst. After an initial rise to a secondary maximum the infrared observations show an exponential drop much like the visual observations. At day 130 after the outburst, however, the $3.7 \mu \mathrm{m}$ data show an excess, which we take as a signature of dust emission. Broadband spectral energy distributions (SEDs) were derived combining published visual photometry and the infrared photometry. The early SEDs during the supernova rise to its secondary maximum could be fitted with blackbodies, but during the exponential decrease the SEDs were too narrow to be fitted by a blackbody energy distribution. The low-resolution spectra were initially featureless, but beyond about day 30 after the outburst, when SN 1993J was in exponential decline, the spectra were dominated by line emission, probably by hydrogen, helium, and iron lines.
\end{abstract}

Key words: supernovae: general — supernovae: individual (SN 1993J)

\section{INTRODUCTION}

Supernova SN 1993J in the galaxy M81 was extremely bright and thus was intensively observed. It was unusual in that its visual light curves and spectra initially showed the characteristics of a hydrogen-dominated Type II supernova, but they soon indicated a transition to a hydrogen-free, helium-dominated supernova, a transition to a so-called Type IIb supernova (Filippenko, Matheson, \& Ho 1993; Swartz et al. 1993). See Filippenko (1997) for a general review of the optical spectra of supernovae.

The visual, radio, and X-ray observations and interpretation are thoroughly discussed in Matheson et al. (2000) and will not be repeated here. Photometric observations of SN $1993 \mathrm{~J}$ in the near-infrared $J$ band $(1.25 \mu \mathrm{m})$ for the two initial months have been reported by Wada \& Ueno (1997), who also list near-infrared observations given in IAU Circulars. Observations by various groups made in the $J, H(1.65$ $\mu \mathrm{m})$, and $K$ bands $(2.2 \mu \mathrm{m})$ during the 50 days after the outburst and reported in IAU Circulars are also referenced by Ray, Singh, \& Sutaria (1993). Very early low-dispersion spectra in the near infrared were reported by Wheeler et al. (1993) and Swartz et al. (1993) who present near-infrared spectra of SN 1993J taken in the $J$ and $K$ bands within a month of the outburst.

Owing to fortuitous scheduling and weather conditions, we were able to obtain near-infrared photometric and spectroscopic observations of SN 1993J during its early development. Because of the singular nature of SN 1993J these unique observations are presented here in their entirety with minimal interpretation, so they will be on record. M81 is at a Cepheid distance of $3.6 \pm 0.3 \mathrm{Mpc}$ (Freedman et al. 1994), which will be used throughout this paper so that $1^{\prime \prime} \simeq 18 \mathrm{pc}$ in projection. Following Lewis et al. (1994) we will adopt the date of the outburst of SN 1993J as 1993 March 27.5 UT (JD 2,449,074).

\footnotetext{
${ }^{1}$ Palomar Observatory, California Institute of Technology, Mail Stop 105-24, Pasadena, CA 91125.

2 SIRTF Science Center, California Institute of Technology, Mail Stop 314-6, Pasadena, CA 91125; kym@caltech.edu, gxn@caltech.edu, lee@ ipac.caltech.edu,bts@mop.caltech.edu.
}

\section{OBSERVATIONS}

Both photometric and spectroscopic observations were made using the near-infrared camera at the Cassegrain $\mathrm{f} / 70$ focus of the Hale 200 inch $(5 \mathrm{~m})$ telescope. The camera had a helium-cooled InSb array with $58 \times 62$ pixels and a plate scale of $0.313^{\prime \prime}$ pixel $^{-1}$ giving a field of $18^{\prime \prime} \times 19^{\prime \prime}$. For both photometric and spectroscopic observations the telescope was guided using an offset guider.

\subsection{Photometry}

Photometric observations were made in the near-infrared atmospheric windows $J, H, K$, and $L^{\prime}$, specified in Table 1 . A "sky" field, usually a field offset to the west by 8 " which contained no obvious sources was interspersed between images of the object. Most of the $L^{\prime}$ observations were made using the chopping secondary mirror with a north-south chop amplitude of $8^{\prime \prime}$.

The photometry of SN 1993J was established relative to the photometric standards set up by Elias et al. (1982), one of which was generally measured just prior to the observation of SN 1993J and one afterward. On five marginally photometric occasions, especially near the outburst, the neighboring star "C" (see, e.g., Benson et al. 1994) was used to obtain relative photometry at $J, H$, and $K$; star C was in turn calibrated against the Elias et al. standards. It has a visual magnitude $V=14.50 \mathrm{mag}$ and visual and nearinfrared colors which are typical of a G-type main-sequence star (Koornneef 1983); its near-infrared magnitudes are included in Table 1. Benson et al. find that in the $V$ band, star $C$ is not variable within an uncertainty of 0.02 mag. In the photometry a synthetic beam of $5^{\prime \prime}$ diameter was used with the local sky taken just outside the object beam. The photometric images were first flat-fielded by using images obtained at twilight.

\subsection{Grism Spectroscopy}

Low-resolution grism observations of SN 1993J, generally accompanying photometric observations, were made on 14 nights spread throughout the observations but sampled more heavily early after the outburst. The dates are given in the figures with the spectra. The slit width was $1^{\prime \prime}$, 
TABLE 1

Photometric ObSERVIng Bands

\begin{tabular}{|c|c|c|c|}
\hline $\begin{array}{l}\text { Central Wavelength } \\
\qquad(\mu \mathrm{m})\end{array}$ & $\begin{array}{c}\text { FWHM }^{\mathrm{a}} \\
(\mu \mathrm{m})\end{array}$ & Band & $\begin{array}{c}\text { Star C Magnitude } \\
\text { (mag) }\end{array}$ \\
\hline 1.27. & 0.25 & $J$ & 13.42 \\
\hline . & 0.32 & $H$ & 13.15 \\
\hline $2.23 \ldots \ldots \ldots \ldots \ldots \ldots$ & 0.40 & $K$ & 13.08 \\
\hline $3.68 \ldots \ldots \ldots \ldots \ldots \ldots \ldots \ldots \ldots$ & 0.64 & $L^{\prime}$ & $\ldots$ \\
\hline
\end{tabular}

a Full width at half maximum of filter.

yielding a resolution $\lambda / \Delta \lambda \approx 80$. Separate spectra were obtained in each of the three near-infrared windows $J(1.19$ $1.33 \mu \mathrm{m}), H(1.54-1.78 \mu \mathrm{m})$, and $K(2.04-2.38 \mu \mathrm{m})$. The grism images in each of the atmospheric windows were reduced separately using standard algorithms. The images were flat-fielded and the atmospheric features canceled by using the chopping secondary mirror of the telescope to sweep a bright $\mathrm{G}$ type star, often HR = BS 3881, in a triangle wave at $50 \mathrm{~Hz}$ along the slit perpendicular to the dispersion direction. The resultant image was assumed to represent the spectrum of a blackbody at the effective temperature of the $\mathrm{G}$ star. The spectra in each wavelength interval were then scaled to the photometry obtained in the appropriate band as given above in order to normalize the three spectra into one spectrum at a common level by integrating the spectral flux densities over the photometric bands and requiring the fluxes to be the same. The flux densities corresponding to $0.0 \mathrm{mag}$ in this normalization are included in Table 2.

\section{RESULTS}

The photometric measurements of SN 1993J, with the dates on which they were obtained, are given in Table 3 and Figure 1. One sigma $(1 \sigma)$ statistical uncertainties are indicated. Systematic uncertainties are probably small and negligible in the $J, H$, and $K$ bands. A good reflection of the systematic uncertainties of the $L^{\prime}$ measurements made in this fairly crowded field is provided by the spread in the measurements made within a few days of each other when the supernova was relatively steady.

The visual observations of SN 1993J were brightest between day 11 and day 34 after the outburst; unfortunately, no Caltech infrared observations were obtained in this critical time. Sporadic observations of the supernova in the $J, H$, and $K$ bands reported in the IAU Circulars and $J$ band measurements of Wada \& Ueno (1997) during this time are included in Figure 1. They tie in with the Caltech observations and mimic the visual light curves, so presumably the infrared curves reached a maximum around day $21, \sim 0.3$ mag brighter than reported in the Caltech observations.
The grism spectra are presented in Figure 2. During the early time after the outburst, the low-resolution spectra were almost featureless, but a week or so after the outburst the spectra became dominated by line emission. On the two occasions where they overlap with the published infrared spectra of Wheeler et al. (1993; $J$ band) and Swartz et al. (1993; $J$ and $K$ bands) the Caltech spectra agree with the previously published spectra.

\section{DISCUSSION}

\subsection{Photometry}

Figure 1 shows that the $J, H$, and $K$ light curves are similar to the visual light curves in exhibiting an early secondary maximum followed by an apparently exponential drop in time. The drop of the $J$ and $H$ bands during the exponential drop-off is significantly steeper than in the visual bands. The colors of SN 1993J, derived directly from the measured magnitudes presented in Figure 1, are given in Figure 3. After an initial sharp increase the $V-K$ and the near-infrared colors are fairly constant. After an initial rise the $V-J$ color becomes significantly bluer than the $V-H$ and $J-H$ or $H-K$ colors. There is a dramatic increase of the $K-L^{\prime}$ color between day 100 and day 130 (Fig. 1), which will be discussed below.

In Figure 4 spectral energy distributions (SEDs) have been created from the visual and near-infrared photometry at the times of the Caltech near-infrared observations. Figure 4 (left) shows SEDs during the initial fall in flux, while Figure 4 (right) shows the SEDs through the subsequent exponential decrease. During the initial sharp fall of the supernova intensity only the visual data obtained within \pm 0.1 days of the infrared observation were included in creating a SED. For day 10 and after day 39, when the change in the supernova emission was more gradual, the data from adjoining days have been averaged into six wider time bins, their width being dictated by the duration in time of close infrared observations; the observations within each bin were concentrated enough in time relative to the changes in emission of the supernova so that an average was considered meaningful. Table 2 gives the magnitude zero points that were assumed in each of the wavebands. The dashed lines in Figure 4 (left) show a blackbody spectrum modified, following Clocchiatti et al. (1995), by extinction (Cardelli, Clayton, \& Mathis 1989), corresponding to $A_{V}=0.74 \mathrm{mag}$. It is seen that the overall SEDs in the early times (less than 50 days) resemble blackbodies with steadily decreasing temperatures. The temperatures derived in Figure 4 (left) are in excellent agreement with those derived by Clocchiatti et al. The values of the temperatures and the extinction do not, however, depend critically on the infrared data. In contrast, in the later days the emission of the supernova was concentrated into a narrower spectral range, the near-infrared emission was depressed relative to a blackbody, and a blackbody fit is impossible (Fig. 4, right).

TABLE 2

Assumed Zero Points

\begin{tabular}{cccccccccc}
\hline \hline Band & $U$ & $B$ & $V$ & $R$ & $I$ & $J$ & $H$ & $K$ & $L^{\prime}$ \\
\hline Zero point $(\mathrm{Jy})^{\mathrm{a}} \ldots$ & 1890 & 4289 & 3709 & 3018 & 2482 & 1578 & 1041 & 646 & 251 \\
\hline
\end{tabular}

a Flux density of $0.0 \mathrm{mag}$. 
TABLE 3

SN 1993J РнотомеTRY

\begin{tabular}{lccccc}
\hline \hline UT Date & Day & $\begin{array}{c}J^{\mathrm{b}} \\
(\mathrm{mag})\end{array}$ & $\begin{array}{c}H^{\mathrm{b}} \\
(\mathrm{mag})\end{array}$ & $\begin{array}{c}K^{\mathrm{b}} \\
(\mathrm{mag})\end{array}$ & $\begin{array}{c}L^{\prime} \\
(\mathrm{mag})\end{array}$ \\
\hline 1993 Mar 30 & 2.8 & 10.76 & 10.75 & 10.74 & $10.74 \pm 0.18$ \\
1993 Mar 31 & 3.7 & 10.58 & 10.53 & 10.49 & $10.36 \pm 0.06$ \\
1993 Apr 01 & 4.7 & 10.63 & 10.50 & 10.40 & $\ldots$ \\
1993 Apr 03 & 6.7 & 10.75 & 10.54 & 10.29 & $\ldots$ \\
1993 Apr 04 & 7.6 & 10.87 & 10.61 & 10.33 & $9.96 \pm 0.04$ \\
1993 Apr 05 & 8.6 & 10.87 & 10.66 & 10.30 & $\ldots$ \\
1993 Apr 06 & 9.9 & 10.98 & 10.67 & 10.32 & $9.64 \pm 0.05$ \\
1993 Apr 07 & 10.9 & 10.85 & 10.61 & 10.29 & $9.77 \pm 0.05$ \\
1993 May 01 & 34.9 & 10.49 & 10.16 & 9.97 & $\ldots$ \\
1993 May 03 & 36.6 & 10.56 & 10.30 & 10.12 & $9.61 \pm 0.05$ \\
1993 May 06 & 39.6 & 10.61 & 10.32 & 10.18 & $9.73 \pm 0.07$ \\
1993 May 07 & 40.6 & 10.61 & 10.32 & 10.16 & $9.69 \pm 0.05$ \\
1993 May 11 & 44.6 & 10.75 & 10.40 & 10.26 & $9.80 \pm 0.05$ \\
1993 Jul 01 & 95.7 & 12.57 & 11.99 & 11.60 & $11.24 \pm 0.08$ \\
1993 Jul 02 & 96.7 & 12.55 & 11.96 & 11.54 & $10.75 \pm 0.09$ \\
1993 Jul 03 & 97.6 & 12.68 & 12.09 & 11.62 & $\ldots$ \\
1993 Jul 04 & 98.6 & $\ldots$ & $\ldots$ & $\ldots$ & $11.06 \pm 0.09$ \\
1993 Aug 04 & 130 & 13.60 & 12.77 & 12.22 & $10.90 \pm 0.10$ \\
1993 Oct 01 & 188 & 14.89 & 14.23 & 13.48 & $11.24 \pm 0.20$ \\
1993 Oct 08 & 195 & 15.10 & 14.37 & 13.65 & $\ldots$ \\
1993 Oct 09 & 196 & $\ldots$ & $\ldots$ & $\ldots$ & $11.70 \pm 0.15$ \\
1993 Nov 25 & 243 & 15.88 & 15.23 & 14.90 & $12.54 \pm 0.12$ \\
1993 Dec 01 & 249 & 16.01 & 15.31 & 14.92 & $11.72 \pm 0.20$ \\
\hline
\end{tabular}

a Day after outburst, which was assumed to be at JD 2,499,074.

${ }^{\text {b }}$ Uncertainty $<0.05$ mag.

Bolometric luminosities of SN 1993J derived from the SEDs in Figure 4 are given in Figure 5. These were obtained by integrating the fluxes in each band in Figure 4 and then adding an additional extrapolation to account for flux beyond the bands noted. Blackbodies at the temperatures indicated in Figure 4 were used to extrapolate the observed fluxes to shorter wavelengths to obtain the bolometric luminosities for the early times (less than day 10). For the later days the flux shortward of the observations was calculated using a linear extrapolation of the observed SEDs. This procedure becomes increasingly uncertain after day 130 and diverges on the final day sampled, so no attempt was made at obtaining a luminosity for that date. The dotted line in Figure 5 shows the mean bolometric luminosity for the first 50 days after outburst derived by Ray et al. (1993), adjusted to the same distance of M81 adopted here. We consider the agreement as good as can be expected given the large extrapolations involved; these extrapolations are especially important in the early times.

After day 100 the emission in the $L^{\prime}$ band showed an excess above an exponential decrease, which we attribute to thermal emission from dust. The existence of the excess is clearly demonstrated in Figures 1 and 3; note, however, that Figure 4 has been plotted to emphasize the wavelengths of the peak emission, and consequently any differences from the exponential fall-off are too small to be seen in that figure.

The excess might possibly be the result of increased line emission as well as thermal emission from dust. In particular, if the $\operatorname{Br} \alpha$ line at $4.051 \mu \mathrm{m}$ were broadened and blue shifted, it could contribute to the $L^{\prime}$ band, which has a long wavelength cutoff of $4.00 \mu \mathrm{m}$. In SN 1987A Meikle et al. (1989) see strong $\operatorname{Br} \alpha$ emission; at its peak up to $40 \%$ of the

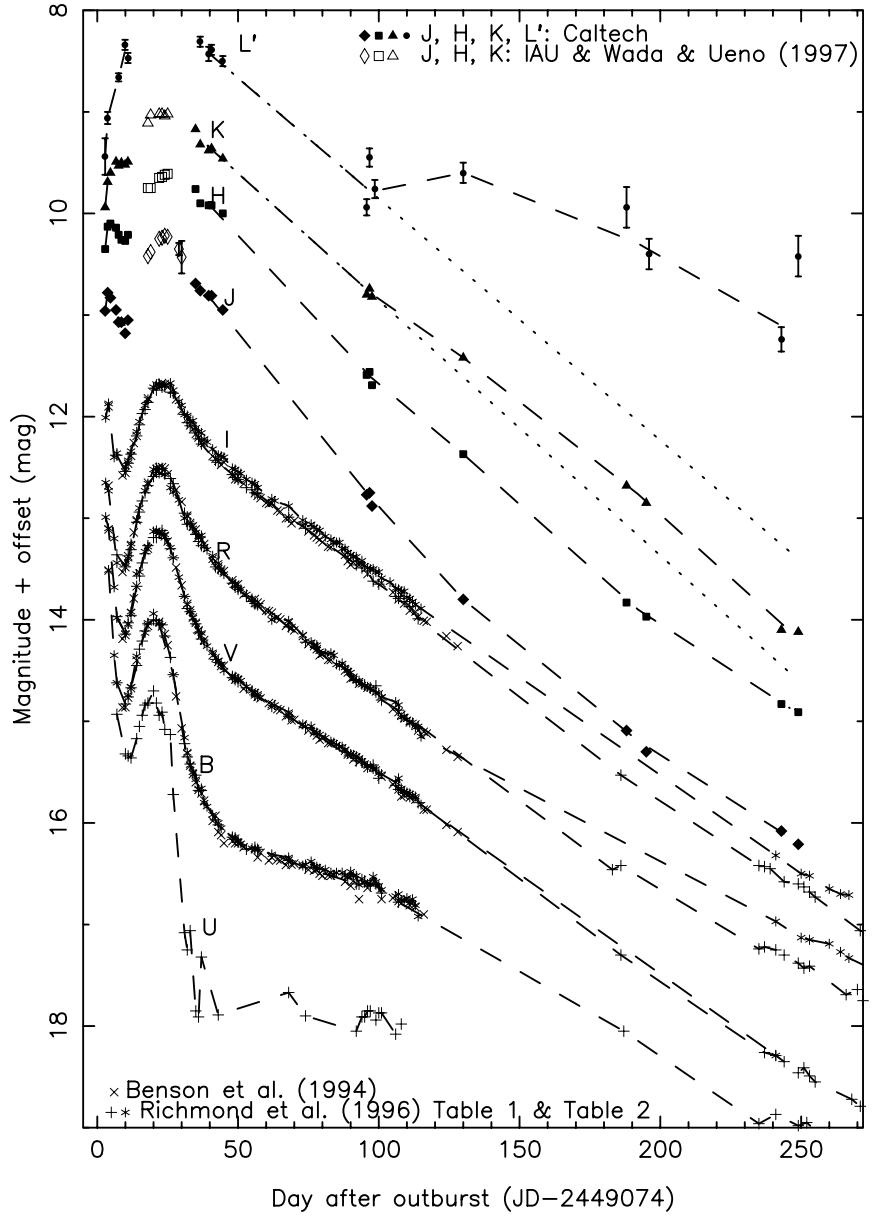

FIG. 1.-Caltech photometry of SN 1993J in the $J, H, K$, and $L^{\prime}$ bands ( filled symbols), along with non-Caltech visual data and occasional $J, H$, and $K$ magnitudes measured near the time of peak emission (open symbols). The magnitude offsets are $+3.5 \mathrm{mag}(U),+2.6 \mathrm{mag}(B),+2.3 \mathrm{mag}(V)$, $+2.0 \mathrm{mag}(R),+1.3 \mathrm{mag}(I),+0.2 \mathrm{mag}(J),-0.4 \mathrm{mag}(H),-0.8 \mathrm{mag}(K)$, and $-1.3 \mathrm{mag}\left(L^{\prime}\right)$. Uncertainties are $1 \sigma$; they are statistical and are plotted only if they exceed $0.05 \mathrm{mag}$; they are discussed in the text. We have adopted the time of outburst as determined by Lewis et al. (1994), i.e., at UT 1993 Mar 27.5 (JD 2,449,074.0). The dashed lines join groups of almost contemporaneous data which have been averaged. The lines are intended only to guide the eye; they do not represent a sophisticated fit to the data. The dotted lines represent the linear extrapolations of the $K$ - and $L^{\prime}$-band data of day 39 and day 97. The visual data are compiled from Benson et al. (1994) and Richmond et al. (1996); the non-Caltech near-infrared data around the peak of the emission curves are taken from Wada \& Ueno (1997) and the IAU Circulars of Lawrence et al. (1993), Romanishin (1993), and Smith (1993).

emission in the $L^{\prime}$ band of SN 1987A could be attributed to line emission. Meikle et al., however, report that a strong $\mathrm{Br} \gamma$ line was present in SN 1987A, while there is no evidence in the spectra of SN 1993J for a $\mathrm{Br} \gamma$ line. As discussed below, the onset of the excess occurred at a time when the strong $\mathrm{P} \beta$ hydrogen line apparently decreased. We will, therefore, attribute the majority of the $L^{\prime}$ excess to thermal emission by dust. If this obtains, some fraction, dependent on the dust temperature, of the $K$-band emission must be contributed by the thermal emission as well.

In order to better characterize the thermal emission, we have assumed that the $K$ and $L^{\prime}$ emission on day 36 and 97 can be identified with photospheric emission in SN 1993J and that the emission greater than the linear extrapolation 

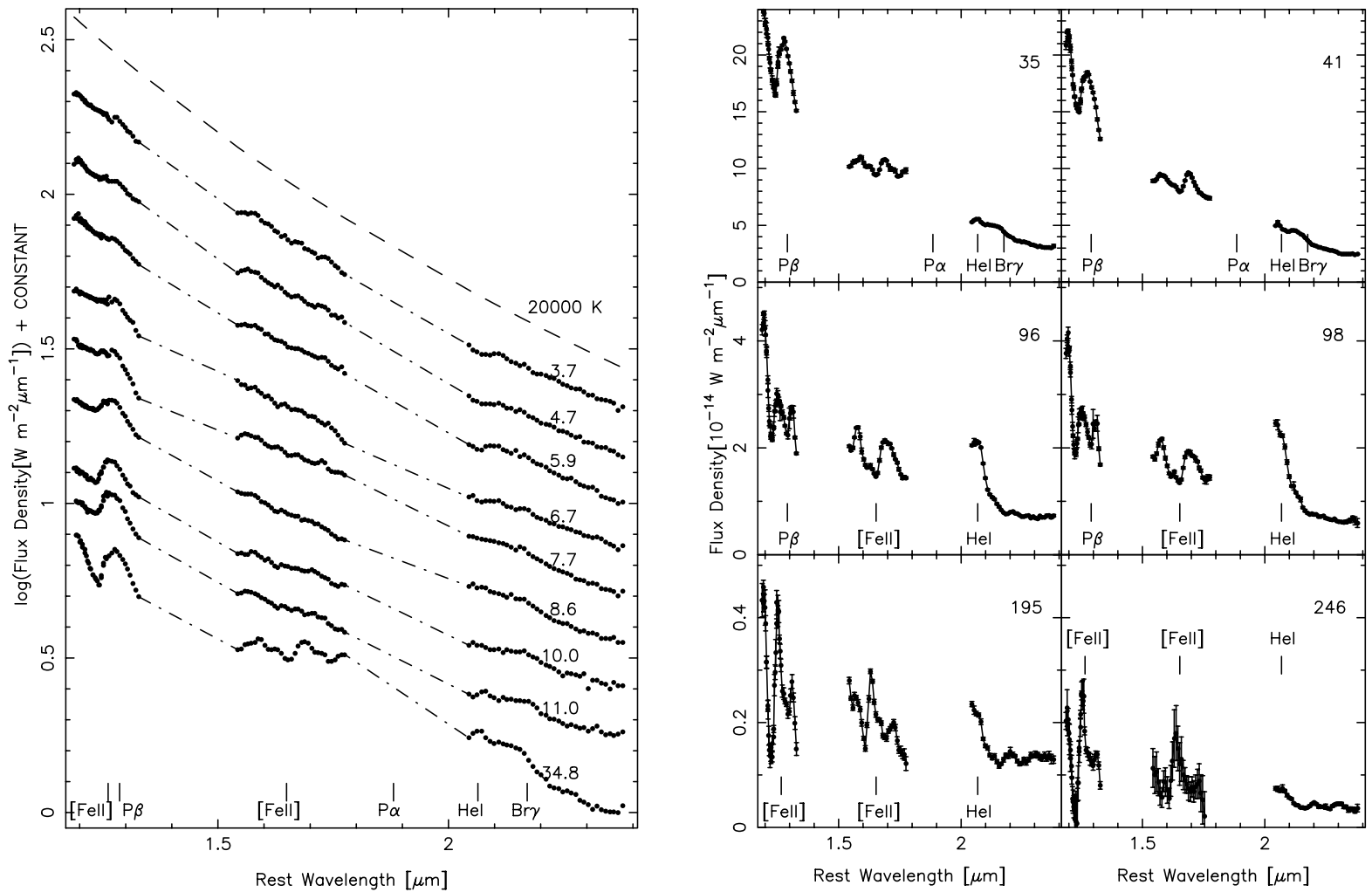

FIG. 2.-Left: Grism spectra of SN 1993J covering the three near-infrared atmospheric windows. Each spectrum is identified by the number of days since the outburst. The dash-dot lines connecting the $J, H$, and $K$ spectra are intended only to guide the eye and do not represent a sophisticated fit to the data. The ordinate is the logarithm of the flux density per wavelength interval; values of the additive constant are 15.01 (day 3.7), 14.82 (day 4.7), 14.66 (day 5.9), 14.47 (day 6.7), 14.34 (day 7.7), 14.17 (day 8.6), 14.00 (day 10.0), 13.84 (day 11.0), and 13.52 (day 34.8). There was no photometry obtained on day 5.9, so the photometry used in normalizing the spectra was interpolated from the previous and next nights. Right: Grism spectra of SN 1993J covering the last six spectra obtained when the supernova was in its exponential decline are shown. Again, each spectrum is identified by the number of days since the outburst. The ordinate is the linear flux density per wavelength interval, each panel is scaled independently, and the spectrum of day 35 is repeated in the two panels to facilitate comparison of the logarithmic and linear displays. The spectrum and photometry labeled day 195 were taken over the 4 days $193-196$. Uncertainties are $1 \sigma$ and are statistical. Prominent lines of hydrogen, helium, and forbidden iron lines are identified at their rest wavelengths; $\mathrm{P} \beta(1.282 \mu \mathrm{m}),[\mathrm{Fe}$ II] (1.257 and 1.644 $\mu \mathrm{m})$, and $\mathrm{He}$ I $(2.059 \mu \mathrm{m})$. Significantly, $\mathrm{Br} \gamma$ was not detected at $2.166 \mu \mathrm{m}$. The wavelength of the $\mathrm{P} \alpha$ line at $1.876 \mu \mathrm{m}$ is indicated, but it is not within the accessible wavelength range of the grism spectra.

of the $K$ and $L^{\prime}$ magnitudes on these days is due to thermal emission by the dust. In Figure 6 we show the excesses in the emission above exponentially decreasing emission at a level smoothly extrapolated from days 36 and 97 . The color temperature implied by the 3.7 and $2.2 \mu \mathrm{m}$ fluxes shown in Figure 6 is $\sim 850 \mathrm{~K}$, declining to $\sim 700 \mathrm{~K}$ during these observations. The color temperature is extremely uncertain. If only half the $K$-band $(2.2 \mu \mathrm{m})$ flux shown were associated with thermal emission from dust, the initial color temperature of the dust thermal emission on day 130 would be reduced to $\sim 700 \mathrm{~K}$. On the other hand, if only half the $L^{\prime}$ band $(3.7 \mu \mathrm{m})$ flux can be attributed to thermal emission from dust, the initial color temperature would be increased to $\sim 1100 \mathrm{~K}$.

As indicated below, iron lines become prominent in the spectra of SN 1993J around the time the dust appears, and it is attractive to assume that the same mechanism (perhaps the shock) heats the gas and the dust. The condensation temperature of iron in the stellar environment is $\gtrsim 1000 \mathrm{~K}$ (Salpeter 1974; J. Wasserburg 2001, private communication). We cannot tell whether the iron was present in the circumstellar medium before the supernova explosion, perhaps as a result of the giant or supergiant phase, or if it was formed in the ejecta at the time of the supernova explo- sion. Gilman (1974) has calculated that spherical iron grains $0.1 \mu \mathrm{m}$ in radius would have an absorption efficiency of 0.15 and from Figure 5, the luminosity of the supernova when the dust was formed was $0.12 \times 10^{35} \mathrm{~W}$. If this central luminosity supplied all the (photon) heating of the dust, grains at $850 \mathrm{~K}$ with an emissivity 0.15 would have to be $8 \times 10^{14}$ $\mathrm{m}$ from the central source. If the grains were silicates or graphite, the predicted radial distance would be larger, but less than doubled; if the grains acted as blackbodies, the predicted radial distance would be smaller. Perhaps coincidentally, VLBI observations of SN 1993J (Marcaide et al. 1995, 1997) have traced a shell-like, apparently almost spherical structure expanding with an expansion speed of $14,900 \mathrm{~km}$ $\mathrm{s}^{-1}$, corresponding to a radial distance of $2 \times 10^{14} \mathrm{~m}$ at 150 days. Marcaide et al. assume that the radio emission comes from a shocked region between the supernova ejecta and the circumstellar material.

The mass of dust can be estimated from $M_{\text {dust }}=\left[f_{\nu}{ }^{*} D^{2}\right] /$ $\left[b_{\nu}\left(T_{\text {dust }}\right)^{*} \kappa\right]$ (e.g., Hildebrand 1983) where $f_{\nu}$ is the observed flux density, $b_{\nu}\left(T_{\text {dust }}\right)$ is the blackbody flux density at a dust temperature of $T_{\text {dust }}, D$ is the distance to the dust shell, and $\kappa$ is the mean opacity of the dust. The mean opacity of carbonaceous interstellar dust at $3.7 \mu \mathrm{m}$ is $\kappa=80 \mathrm{~m}^{2} \mathrm{~kg}^{-1}$ ( $\mathrm{Li}$ $\&$ Draine 2001). If the dust grains have a similar opacity and 


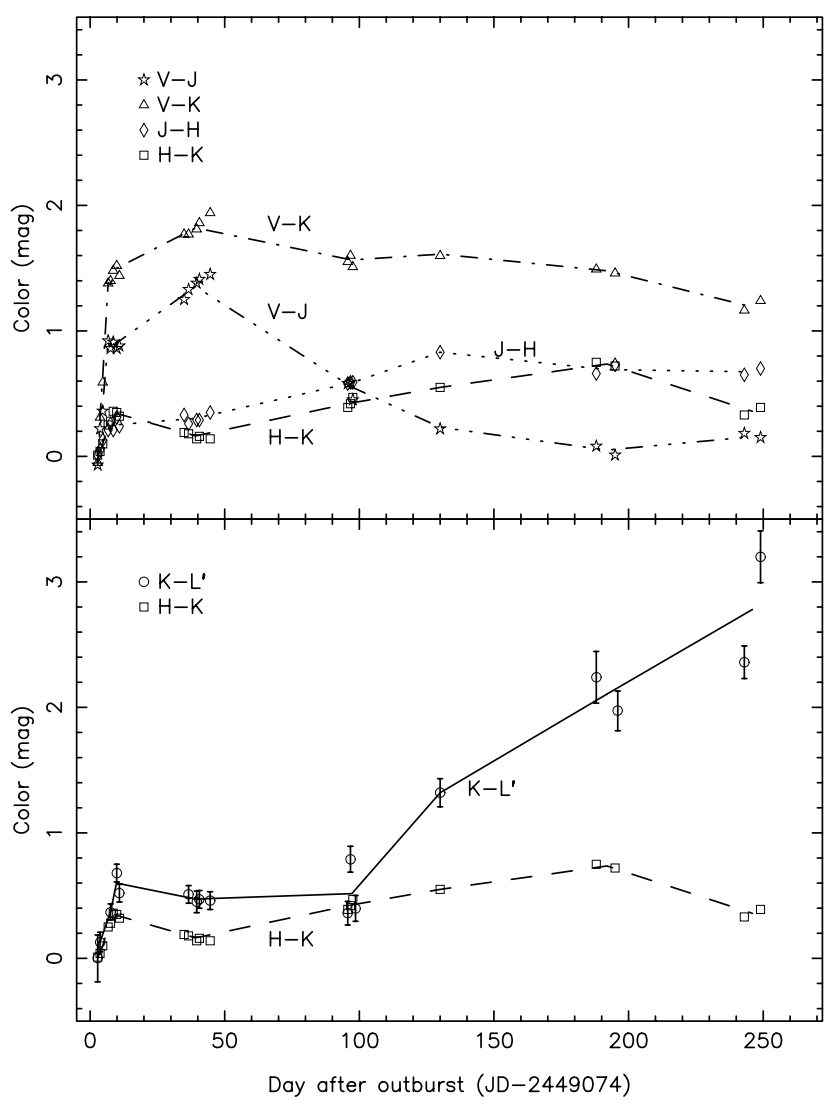

FIG. 3.-Near-infrared colors determined from the observed magnitudes of SN $1993 \mathrm{~J}$ along with the derived $V-K$ and $V-J$ colors. The blue color of the $V-J$ curve relative, for example, to the $V-K$ curve during the exponential drop off of the supernova emission is evident in the top panel as is the excess in the $L^{\prime}$ band, attributed to thermal emission from dust, in the lower panel. The $H-K$ color is repeated in both panels to ease comparison. For the $V$ band, data were taken from Benson et al. (1994) or Richmond et al. (1996). When there was no $V$-band measurement made on the same day as the near-infrared measurement, the visual data were interpolated between neighboring visual measurements. As in Fig. 1, lines are drawn between clumps of data, are intended only to guide the eye, and do not represent a sophisticated fit to the data. Uncertainties are $1 \sigma$ and reflect only the statistical uncertainties.

are at a grain temperature equaling their color temperature, i.e., at $T_{\text {dust }} \sim 850 \mathrm{~K}, M_{\text {dust }}=\sim 10^{-5} M_{\odot}$.

The excess in the $L^{\prime}$ band is not unprecedented. Meikle et al. (1993) describe a very similar excess commencing at day 350 in the Type II SN 1987A, which they ascribe to more than $3 \times 10^{-4} M_{\odot}$ of dust probably having a clumped distribution. They find, from more extensive data than available here for SN 1993J, a color temperature decreasing from $\sim 700 \mathrm{~K}$ and an average expansion velocity $\sim 2300 \mathrm{~km} \mathrm{~s}^{-1}$. The conjecture that dust was present in SN 1987A is directly confirmed by the mid-infrared observations of Roche, Aitken, \& Smith (1993) and Wooden et al. (1993). In the Type IIn SN 1998S Fassia et al. (2000) report a similar high excess in the $L^{\prime}$ band present at day 130 , which they also attribute to thermal emission from dust.

\subsection{Low-Resolution Spectroscopy}

During the very early time after the outburst, the low-resolution spectra were almost featureless, and, as is shown by the dashed curve in Figure $2(l e f t)$, the continua in the early spectra closely followed a hot blackbody. The wavelength range was restricted to the Rayleigh-Jeans portion of the spectrum and the spectra thus do not set other than a lower limit on the temperature. When the supernova started its exponential decline in emission, the near-infrared spectra changed from a smooth continuum to become dominated by line emission, and at approximately the same time the SED changed from nearly a blackbody SED to a much narrower SED.

The line identification in the near-infrared spectra is complicated by the low resolution of the spectra as well as the fact that the level of the continuum is uncertain. In addition, lines are blended, potentially show P Cygni profiles, and have different broadening and different blue shifts relative to the systemic velocity of M81, depending on the time after the outburst and the optical depth at which they were formed. The peak observed at $1.28 \mu \mathrm{m}$, seen clearly as early as day 6.7 and present through day 98 , was already marginally visible on day 3.7 and can be identified with blue shifted $\mathrm{P} \beta\left(\lambda_{\text {rest }}=1.282 \mu \mathrm{m}\right)$. Unfortunately, $\mathrm{P} \alpha\left(\lambda_{\text {rest }}=1.876 \mu \mathrm{m}\right)$ fell in a wavelength region where the atmosphere is opaque. Significantly, $\operatorname{Br} \gamma\left(\lambda_{\text {rest }}=2.167 \mu \mathrm{m}\right)$ was not detected, although it falls in a relatively clear portion of the spectrum. [Fe II] lines at $\lambda_{\text {rest }}=1.257$ and $\lambda_{\text {rest }}=1.644 \mu \mathrm{m}$ were prominent, again blue shifted, at 1.252 and $1.633 \mu \mathrm{m}$ on days 195 and 246. The $1.644 \mu \mathrm{m}$ line, which was in a clear portion of the spectrum, first appeared faintly on day 98 . The mass in [Fe II] can be estimated from the observed line flux on day $195,2.2 \times 10^{-17} \mathrm{~W} \mathrm{~m}^{-2}$, and the Einstein A coefficient, $1.9 \times 10^{-3} \mathrm{~s}^{-1}$, to imply that greater than $10^{-4} M_{\odot}$ was present in [Fe II]. The He I line at $\lambda_{\text {rest }}=2.059 \mu \mathrm{m}$ was out of the wavelength range of the spectrometer but was strong enough and broadened enough that, although it was obviously blue shifted, its effects were noticeable in the spectra after day 41 . Features at $\lambda=1.20,1.31,1.69 \mu \mathrm{m}$ seem clear emission peaks but could not be readily identified. Swartz et al. (1993) suggest the feature at the edge of the spectra at $1.19 \mu \mathrm{m}$ could be Ca II lines at $\lambda_{\text {rest }}=1.184$ and $1.195 \mu \mathrm{m}$. Meikle et al. (1993) identify a line at $1.203 \mu \mathrm{m}$ in SN 1987A with Si I at $\lambda_{\text {rest }}=1.198 \mu \mathrm{m}$. An interesting alternative is to identify the feature with the lines of [Ni II] shortward of 1.19 $\mu \mathrm{m}$ (Quinet \& Le Dourneuf 1996; Henry 1987).

The mean wavelength shift of the two [Fe II] lines on day 195, the sharpest lines in these spectra, corresponds to a velocity of $\sim 1600 \mathrm{~km} \mathrm{~s}^{-1}$ blue shifted with respect to the systemic velocity of M81. This is not atypical of line velocities found in other supernovae, but it is significantly smaller than the expansion velocity measured in the VLBI data (Marcaide et al. 1995, 1997). Even at the low resolution of the spectra many of the lines were resolved. For example, the $\mathrm{P} \beta$ line $\sim 10$ days after the outburst had an intrinsic full width at half maximum of about $0.04 \mu \mathrm{m}$ or $10,000 \mathrm{~km} \mathrm{~s}^{-1}$.

The behavior of the spectra of SN 1993J presented here is very similar to that shown by the near-infrared spectra of SN 1987A of Meikle et al. $(1989,1993)$ although the timescale in SN 1987A is significantly longer than that in SN 1993J. Specifically, in the early days after the outburst (days $\lesssim 100$ ) the near-infrared spectra of SN 1987A were also essentially featureless (Meikle et al. 1989). After that, the spectra of SN 1987A were dominated by line emission, giving an appearance much like that of the SN 1993J spectra. In fact, with the higher spectral resolution $(\lambda / \Delta \lambda \simeq 400$ 

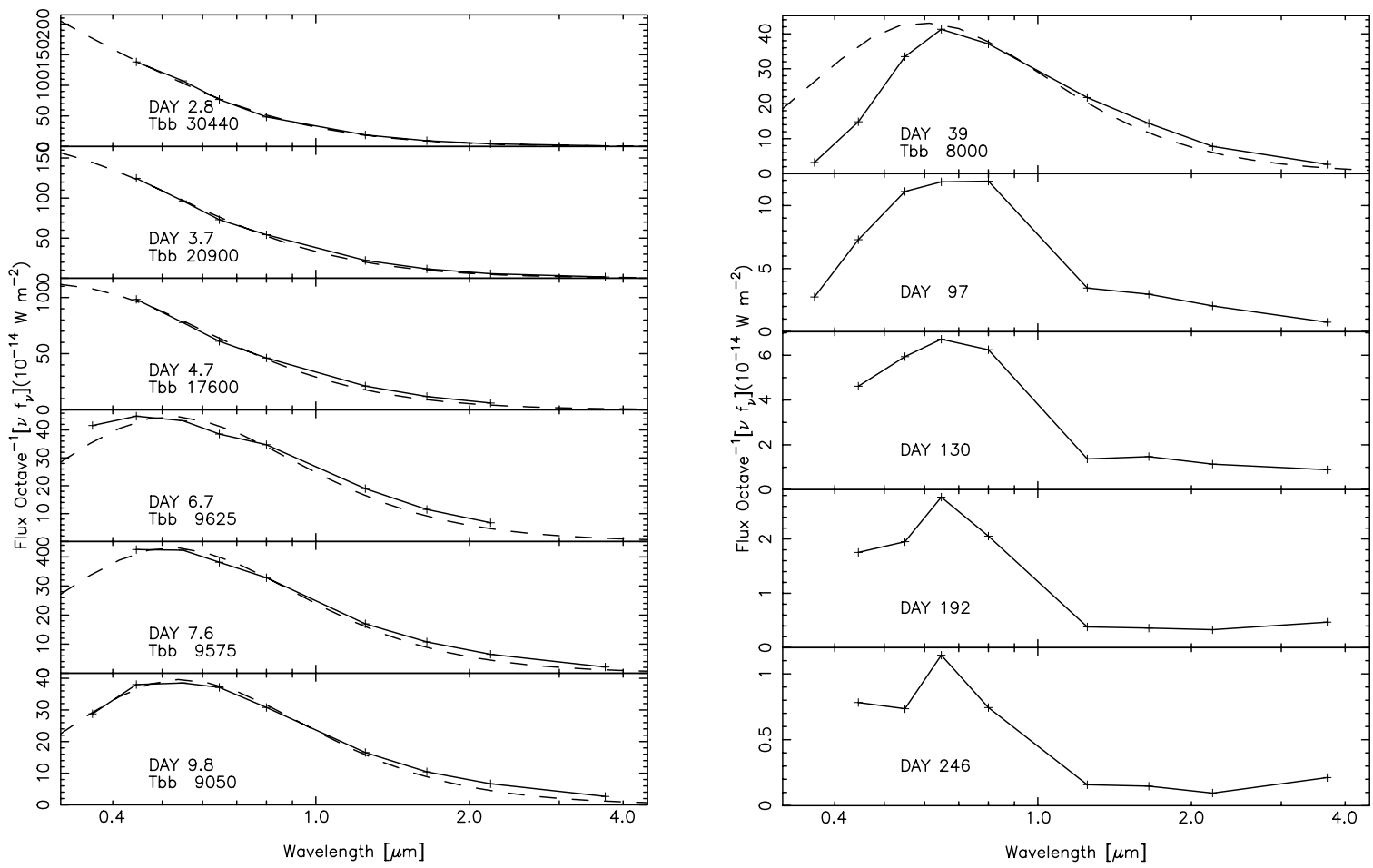

FIG. 4. - Left: Spectral energy distributions (SEDs) of SN 1993J during its initial fall in flux and calculated at the times of the Caltech infrared measurements are shown. The SEDs for times less than 9.6 days after the outburst use the Caltech infrared observations combined with visual observations taken within \pm 0.1 day of the infrared observations; some visual observations therefore had to be interpolated. Blackbody curves at the temperatures indicated with an extinction of $A_{V}=0.74 \mathrm{mag}$ and scaled to the observed data are shown by dashed lines. The differences squared between the blackbody curves and the SEDs for the $B, V$, $R, I, J, H, K$, and $L^{\prime}$ bands were minimized to determine the blackbody temperature. Right: SEDs calculated at the times of the Caltech infrared measurements are shown. For these times, several observations have been averaged into bins, governed by the timing of the infrared observations, during which the change in the supernova flux curve made such averages meaningful. The blackbody curve on the top panel (dashed curve) has been normalized to the SEDs at $R, I, J, H$, $K$, and $L^{\prime}$ and is included mainly to illustrate the fact that a blackbody curve is too wide to fit the SEDs at later times after the outburst.

and 1000), the line identifications in the SN 1987A spectra of Meikle et al. serve as a line identification source list for SN 1993J and, more importantly, serve to emphasize the real complexity inherent in the near-infrared spectra of supernovae.

Although the timescales are different, there is a, perhaps superficial, resemblance between both the near-infrared spectral development and the $3.7 \mu \mathrm{m}$ photometric appearance of SN 1993J and SN 1987A. Clearly the infrared properties of supernovae are important for their evolution and must be incorporated into any final description of the supernovae and their role in enriching and heating the interstellar medium.

We thank the staff of the Palomar Observatory for their assistance in making these observations possible. We thank S. E. Persson who let us observe SN 1993J during some of his regularly scheduled observing time. We thank A. Filippenko and T. Matheson for providing us with information about this object; they and J. Graham read an early draft of the paper and provided helpful comments. J. Cohen, E. Egami, N. Scoville, and J. Wasserburg made valuable comments, and M. Neugebauer helped prepare this paper. Thank you. B. T. S., G. N., and K. M. were supported by grants from the NSF and NASA. L. A. and B. T. S. are supported by the SIRTF Science Center at Caltech. SIRTF is carried out at JPL, operated by Caltech under an agreement with NASA.

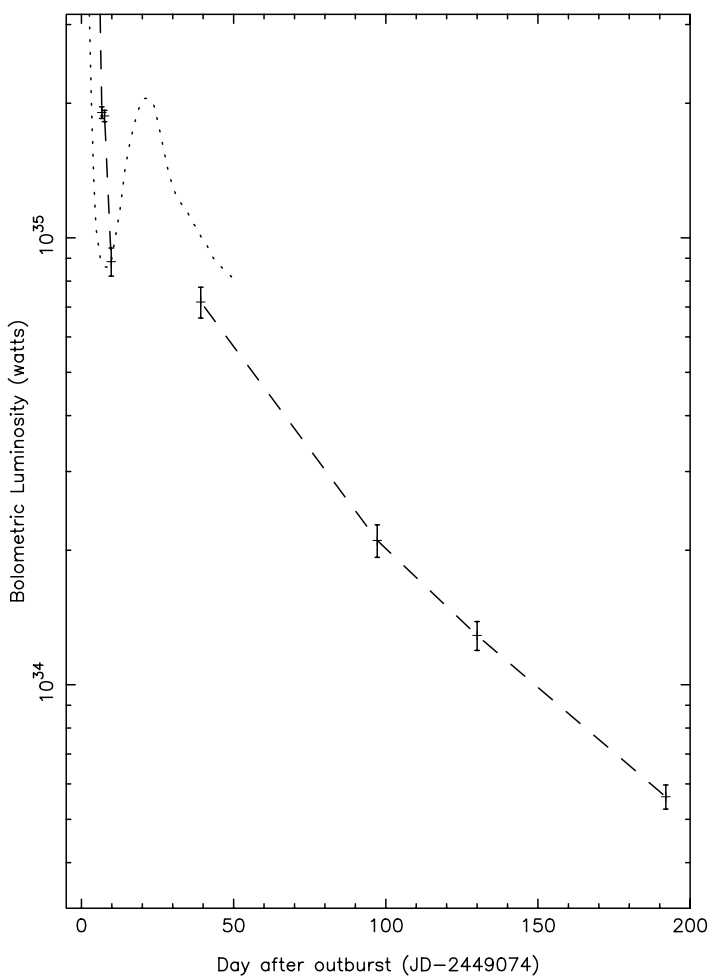

FIG. 5.-Bolometric luminosities derived by integrating the SEDs of Fig. 4. The dashed lines are intended only to guide the eye. The dotted curve is a mean taken by eye from the bolometric luminosities derived by Ray, Singh, \& Sutaria (1993). The SEDs of the early time (less than 10 days) were extrapolated using the blackbody curves shown in Fig. 4 to obtain bolomet- 


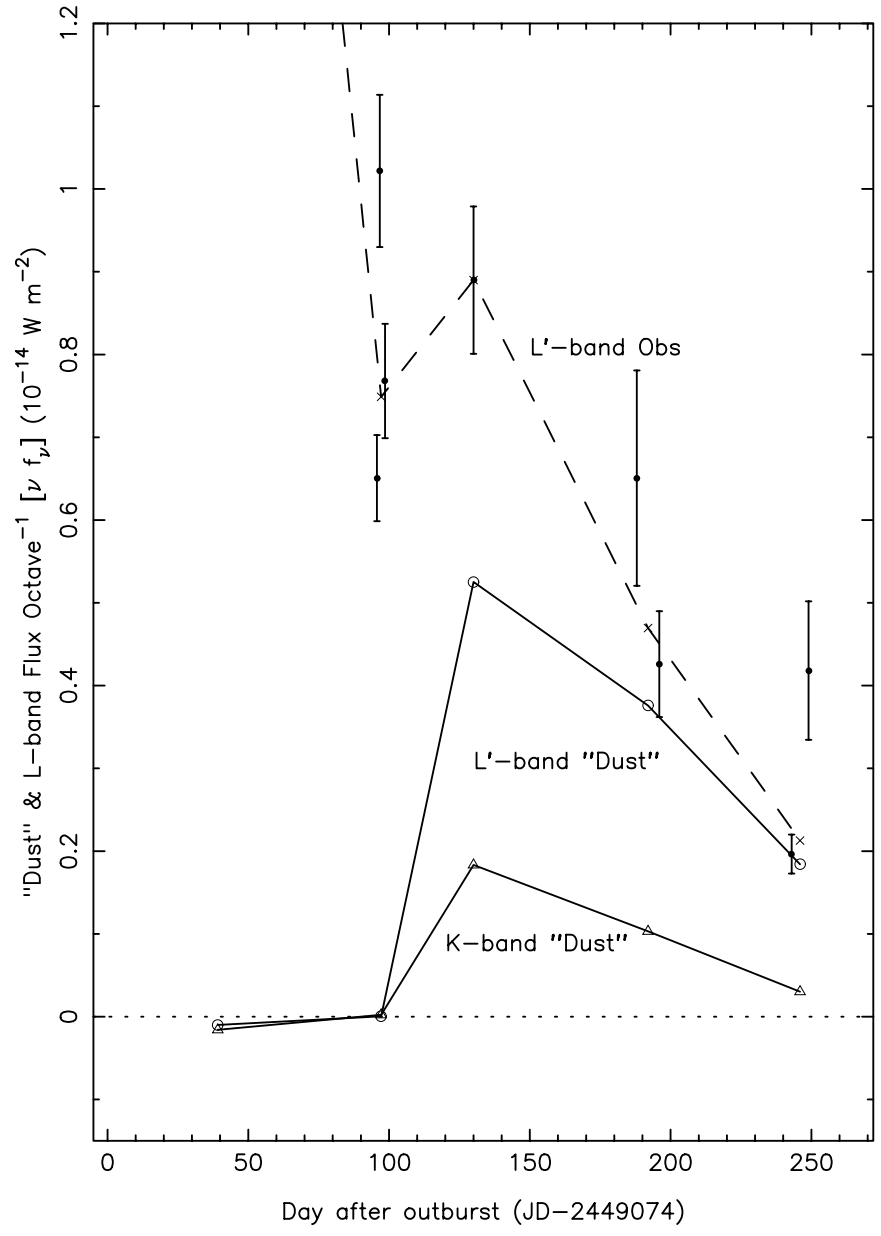

FIG. 6.-The excesses above an exponentially falling flux are shown by open symbols, as well as the total measured flux per octave in the $L^{\prime}$ band represented by the closed circles. The solid lines correspond to the excess, the putative "dust" component in SN 1993J: the open circles represent the $L^{\prime}$ band and the open triangles the $K$ band. The baseline exponential, represented by the dotted line, has been set by the data of day 36 and day 97 as described in the text and shown in Fig. 1. The x's mark the times and average flux per octave observed in four of the bins of data described in the caption to Fig. 1. Uncertainties are $1 \sigma$ and are statistical.

Benson, P. J., et al. 1994, AJ, 107, 1453

Cardelli, J. A., Clayton, G. C., \& Mathis, J. S. 1989, ApJ, 345, 245

Clocchiatti, A., Wheeler, J. C., Barker, E. S., Filippenko, A. V., Matheson, T., \& Liebert, J. W. 1995, ApJ, 446, 167

Elias, J. H., Frogel, J. A., Matthews, K., \& Neugebauer, G. 1982, AJ, 87, 1029

Fassia, A., et al. 2000, MNRAS, 318, 1093

Filippenko, A. V. 1997, ARA\&A, 35, 309

Filippenko, A. V., Matheson, T., \& Ho, L. C. 1993, ApJ, 415, L103

Freedman, W. L., et al. 1994, ApJ, 427, 628

Gilman, R. C. 1974, ApJS, 28, 397

Henry, R. B. C. 1987, ApJ, 322, 399

Hildebrand, R. H. 1983, QJRAS, 24, 267

Koornneef, J. 1983, A\&A, 128, 84

Lawrence, G. F., Paulson, A., Mason, C., Butenhoff, C., \& Gehrz, R. D. 1993, IAU Circ. 5844

Lewis, J. R., et al. 1994, MNRAS, 266, L27

Li, A., \& Draine, B. T. 2001, ApJ, 554, 778

Marcaide, J. M., et al. 1995, Science, 270, 1475 . 1997, ApJ, 486, L31

\section{REFERENCES}

Matheson, T., et al. 2000, AJ, 120, 1487

Meikle, W. P. S., Allen, D. A., Spyromilio, J. \& Varani, G.-F. 1989, MNRAS, 238, 193

Meikle, W. P. S., Spyromilio, J., Allen, D. A., Varani, G.-F., \& Cumming, R. J. 1993, MNRAS, 261, 535

Quinet, P., \& Le Dourneuf, M. 1996, A\&AS, 119, 99

Ray, A., Singh, K. P., \& Sutaria, F. K. 1993, JApA, 14, 53

Richmond, M. W., Treffers, R. R., Filippenko, A. V., \& Paik, Y. 1996, AJ, 112,732

Roche, P. F., Aitken, D. K., \& Smith, C. H. 1993, MNRAS, 261, 522

Romanishin, W. 1993, IAU Circ. 5773

Salpeter, E. E. 1974, ApJ, 193, 579

Smith, J. A. 1993, IAU Circ. 5780

Swartz, D. A., Clocchiatti, A., Benjamin, R., Lester, D. F., \& Wheeler, J. C. 1993 , Nature, 365,232

Wada, T., \& Ueno, M. 1997, AJ, 113, 231

Wheeler, J. C., et al. 1993, ApJ, 417, L71

Wooden, D. H., Rank, D. M., Bregman, J. D., Witteborn, F. C., Tielens, A. G. G. M., Cohen, M., Pinto, P. A. \& Axelrod, T. S. 1993, ApJS, 88, 477 